\title{
INTELLIGENT COMPUTING TECHNIQUES ON MEDICAL IMAGE SEGMENTATION AND ANALYSIS: A SURVEY
}

\author{
Daizy Deb ${ }^{1}$, Sudipta Roy ${ }^{2}$ \\ ${ }^{1}$ Information Technology Department, Assam University, Silchar \\ ${ }^{2}$ Information Technology Department, Assam University, Silchar
}

\begin{abstract}
Today, a wide range of different image segmentation techniques can be found in medical imaging. Image segmentation is an important part of image processing, which often has a large impact on quantitative image analysis results. These allow the radiologist to obtain two and three-dimensional view of the internal structures of the human body, it has a large application domain for redundant clinical problems. Hence this paper gives insight into the various literatures published for the last decades on medical image segmentation and texture detection, its applicability into various domains of image processing and to identifying the further research problems.
\end{abstract}

Keywords-Segmentation, Soft Computing, Medical Imaging, Magnetic Resonance, Colonography

\section{INTRODUCTION}

Image segmentation is a important factor of computer vision. It is the image pixel into meaningful group so that we can achieve a compact representation on the image. Medical imaging is the technique and process used to create images of the human body for clinical purposes (medical procedures seeking to reveal, diagnose, or examine disease) or medical science . Measurement and recording techniques which are not primarily designed to produce images, such as electroencephalography (EEG) , magneto encephalography (MEG),electrocardiography (EKG), and others, but which produce data susceptible to be represented as maps (i.e., containing positional information), can be seen as forms of me image segmentation is the process of partitioning a digital image into multiple segments (sets of pixels, also known as super pixels).The goal of segmentation is to simplify and/or change the representation of an image into something that is more meaningful and easier to analyze clinical imaging. The result of image segmentation is a set of segments that collectively cover the entire image, or a set of contours extracted from the image (see edge detection). Each of the pixels in a region are similar with respect to some characteristic or computed property, such as color, intensity, or texture. Adjacent regions are significantly different with respect to the same characteristic(s). When applied to a stack of images, typical in medical imaging, the resulting contours after image segmentation can be used to create $3 \mathrm{D}$ reconstructions with the help of interpolation algorithms.

The different types of segmentations are: (i) Pixel-Based segmentation, (ii) Edge-Based segmentation , (iii) Region-based segmentation , (iv) Model-based segmentation , (v)Color Image Segmentation, (vi) Gray-scale Image Segmentation, (vii) Text
Segmentation, (viii) Unsupervised segmentation , Supervised segmentation[10].

This paper aims to make a review on the current segmentation algorithms used for medical images. Algorithms are classified according to their principal methodologies, namely the ones based on thresholds, the ones based on clustering techniques and the ones based on deformable models.

In the first phase of this paper discuss about the paper that last type is focused on due to the intensive investigations into the deformable models that have been done in the last few decades. Typical algorithms of each type are discussed and the main ideas, application fields, advantages and disadvantages of each type are summarized. In the second phase, image segmentation based on softcomputing techniques for unsupervised learning in medical imaging.

Medical image segmentation which is the process of outlining relevant anatomical structures in an image dataset is a problem that is central to a variety of medical applications including image enhancement and reconstruction, surgical planning, disease classification, data storage and compression, and 3Dimensional (3D) visualization. Some of the methods used for medical image segmentation fall under deformable models. The use of deformable model was popularized by Kass et al. (1990), snake model was used to develop active contour model that minimizes energy functional under the influence of forces which are internal force, image force and external constraint force. Deformable models can be classified into three categories which are:

(i) Free-form,

(ii) Parametric and 
(iii) Geometric active contour model.

\section{OVERVIEW OF 3D IMAGE SEGMENTATION}

Researchers at the Swiss Federal Institute of Technology in Lausanne, Switzerland have developed many applications for viewing the images in the Visible Human dataset. The Visible Human Slice Sequence Animation Web Server is a web based service for extracting slices, curved surfaces and slice animations from the Visible Human dataset [1]. The application also allows users to construct 3D anatomical scenes using combinations of slices from the dataset and 3D models of internal structures, and extract 3D animations.

The real-time interactive visible human navigator was also developed at the Swiss Federal Institute of Technology [2]. This application has the ability to extract arbitrarily oriented and positioned slices from the Visible Human dataset in real-time. A continuous sequence of slices is displayed in real-time in response to navigation commands from the user. The NPAC Visible Human Viewer [3] developed at Syracuse University, was the first online viewer developed for visualizing the VHP dataset. This Java applet allows users to select and view twodimensional images of the human body from 3 different angles. There have been many enhancements to the viewer since its debut in 1995.

To create a three-dimensional model of the human body, researches at the University of Geneva developed the Comprehensive Human Animation Resource Model (CHARM) [4]. The CHARM project is an attempt at providing a structured and dynamic model of the body in contrast to the unstructured and static data provided by the Visible Human dataset. Twodimensional contours are segmented from anatomical and CT images to form 3D surfaces of the upper left limb. CHARM includes a labeling tool for identifying each organ of interest in the $2 \mathrm{D}$ images.

\section{COMPARISON BETWEEN SOME MODEL- BASED SEGMENTATION}

The human vision system has the ability to recognize objects even if they are not completely represented. specific knowledge about the geometrical shape of the objects is required, which can then be compared with the local information. This train of thought leads to model-based segmentation.

Shape constraint is of special importance in medical applications, where prior knowledge of the anatomy is needed to help constrain the evolution of the segmentation process.

Deformable models [3] are physics-based models that are capable of controlling the geometry and smoothness of the segmented boundaries, and allowing significant variability of the biological structures. In deformable model formulations, two energy terms are considered to evolve the contour.
(i)The external energy term is obtained from the image information, such as intensity level and intensity gradient.

(ii) The internal energy term is from the contour smoothness.

Lower energy corresponds to smaller curvature and smaller change in curvature. Thus deformable model segmentation is formulated as an energy minimization problem.

In this method, designed a tube-shaped shape model to direct the segmentation and tracking. Voxel intensities and intensity gradients in the local region are the main image forces considered. Before the segmentation begins, a seed point in the lumen area needs to be selected manually. Then try to fit a initial cylinder (tube) model for this seed position in an initialization step. Then the initialized model should be able to grow in both directions and track over the colon, allowing possible tuning of the parameters of the shape model. The shape model is designed as a bendable cylinder, which is controlled by 5 parameters, such two coordinate values of translation, two coordinate values of rotation angles and radius from the center of the tube [7].

3D shape modeling and tracking approach for segmentation in tube-shaped structures and its implementation in colonography using Adaboost Process.
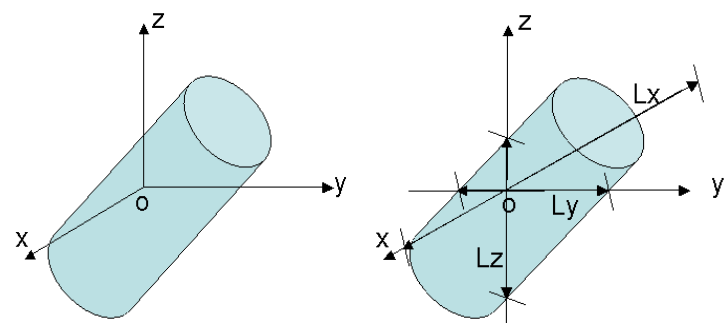

Fig 1 (a) the tube-shaped model is determined by 3 parameters scheme.

From these 5 tunable parameters, Gaussian distributions can determine.

Suppose Ei and Ee are energy values from the intensity and edge terms, and to minimize the both energies.

Then the combined energy function is set as:

$$
\mathrm{E}=\mathrm{e}^{\mathrm{k}(\mathrm{Ei}-\mathrm{Eip})}+\mathrm{e}^{\mathrm{Ee}-\mathrm{Eep}}
$$

Where Eip and Eep are the energy values from the previous step.

The combined energy value $\mathrm{E}$ gives a performance measurement of the tracking. If $\mathrm{E}$ keeps less than or equal 2, we can say the tracking process is not getting worse. Since random sampling is not efficient, we try to iteratively shift the sampling center and narrower the distribution range if $\mathrm{E}$ is less than a 
certain threshold. In this way our sampling method converges much faster.
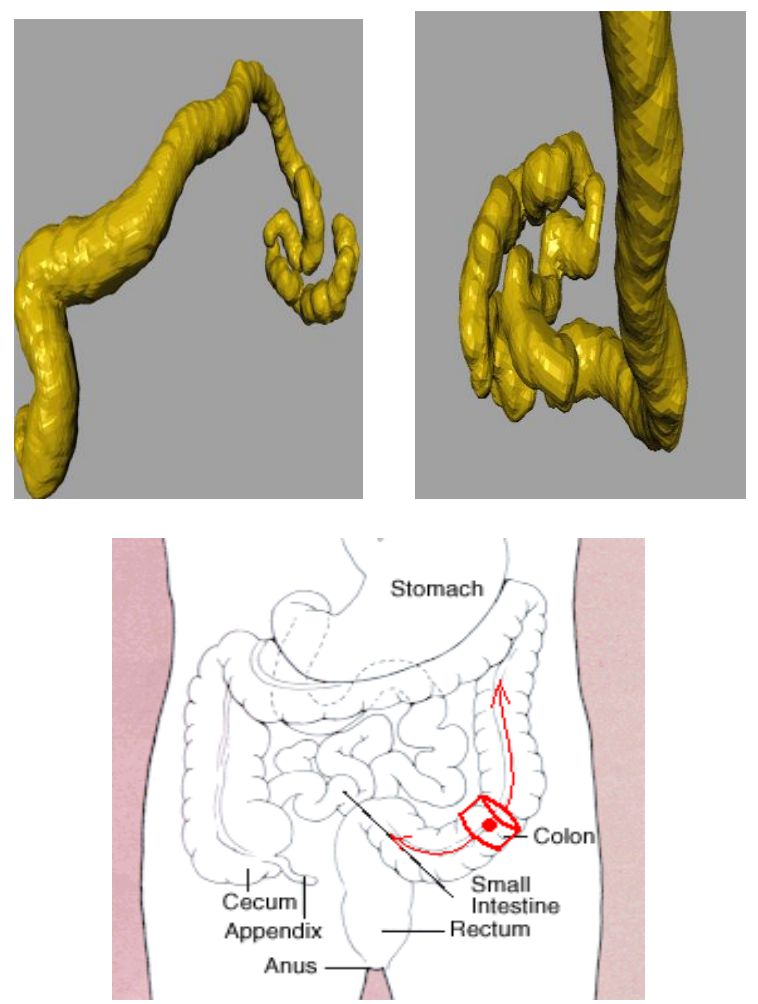

Fig 2 A result of the tracking method in two view angles. The colon portion segmented is the right hand side part of a patient's colon.

Many shape modeling methods use a linear combination of the component vectors to represent the possible variations given the input training shapes. For example active shape model were first introduced by T. F. Cootes and C.J. In their ASM algorithm [6], a statistic shape model is set up based on the principle component analysis (PCA) of a set of training data. PCA method uses a small set of principle components to represent a large data set. And a shape can be reconstructed by a linear combination of the mean shape and a weighted sum of the principle components. There are also some other shape modeling methods such as independent component analysis (ICA) and factor analysis (FA) which are basically similar to the PCA method. However, all these methods have limitations. In practice, the input training data may not align well and are usually subjected to random transformations, such as translation, rotation, and scaling.. B. Frey and N. Jojic proposed the transformed component analysis (TCA) method in 1999 [6]. They set up a mixture model to jointly estimate the image components and the spatial transformations, such as translation, scaling, rotation and shearing.

Due to the noisy and complex nature of tagged MR images, without the use of prior knowledge, accurate boundary tracking based solely on the MR images is usually not possible. So it use the expert's manual contours as the training input. There is a set of points to describe a shape. That is, in each input image, we have to choose a set of landmark points on the object contours to represent the object's shape. These landmark points should be consistently located from one input image to another. In practice, this landmark choosing process would be very time consuming. Those concept of landmark has been implemented on segmentation using Cardiac Tagged MRI.

Since the shape of the mid portion of the heart in short axis (SA) images is consistent and topologically fixed (one left ventricle (LV) and one right ventricle (RV)), it is reasonable to implement an active shape model [5] to represent the desired boundary contours. Then a few feature points are selected automatically using geometry features such as maximum curvature, and all the other landmark points are chosen automatically by equally spacing them between feature points. In this application, the SA contours are automatically discretized into 50 ordered landmark points. Then the coordinates of the 50 landmark points are reshaped to a 100 element vector $\mathrm{X}$, where,

$$
\mathrm{X}=(\mathrm{x} 1, \mathrm{y} 1, \mathrm{x} 2, \mathrm{y} 2, \ldots, \mathrm{x} 50, \mathrm{y} 50) \mathrm{T} \ldots \ldots(\mathrm{i})
$$

Given s training examples, we generate s such vectors $\mathrm{xj}$. Then we perform TCA on these vectors.

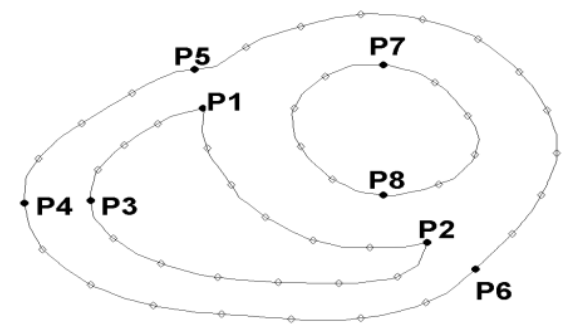

Fig: 3 The illustration of the method used to place the landmark points.

Allowing possible rotation transformations, $\mathrm{X}$ can be approximately represented by $\mathrm{R} \cdot(\mu+\mathrm{Pb})$, where $\mathrm{R}$ is a rotation matrix, $\mu$ is the mean shape, $\mathrm{P}$ is the shape variation components matrix, and $\mathrm{b}$ is the component parameter vector, which determines a subspace representation of the shape $\mathrm{X}$.

This is a probability model that jointly estimates the rotation matrix and the component model $\mathrm{P}$ and $\mathrm{b}$ via an EM approach. The goal of the EM algorithm is to maximize the distribution over the training shape, the rotation angle, the latent shape and the component model.

Then applied this rotation invariant shape modeling method to some real heart shape data, which arterially added some rotation distortion to so that make the results more obvious. The following is the input training data set. They are translated and 
scaled before the training process. We can find they have different orientations:

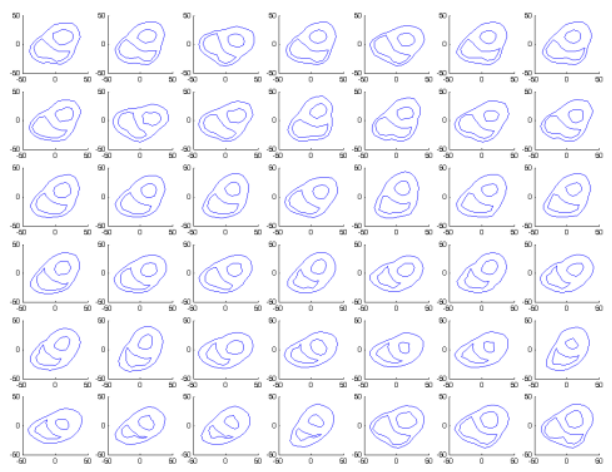

Fig 4 From that we can generate some simulated heart shapes with the help of PCA and TCA .

For 2D tagged MRI, we learn the shape and local appearance model from a training set. This shape model is a linear rotation invariant model, on which each control point has its own local appearance model. This appearance model is learned from an Adaboost process, which integrates many features. In each application, besides the models, we also give complete details in solving the segmentation problems, such as how we correct the MR image intensity inhomogeneity and how we automatically initialize the segmentation.

The limitation of that model is In the 2D cardiac tagged MRI segmentation, the size of training data set is critical for the segmentation performance, since all the models are obtained from learning. We find that if the segmentation method is applied to images at phases or positions that are not represented in the training data, the segmentation process tends to get stuck in local minima. Thus the training data need to be of sufficient size to cover all possible variations that may be encountered in practice.

Enhanced Geometric Active Contour Segmentation Model (ENGAC): Active contour (GAC) segmentation which is one of the outstanding model used in machine learning community to solve the problem of medical image segmentation. However, GAC has problem of deviation from the true outline of the target feature and it generates spurious edge caused by noise that normally stop the evolution of the surface to be extracted. In this paper, an enhanced Geometric active contour was formulated by hybridizing Kernel Principal Component Analysis(KPCA) with the existing Geometric active contour segmentation model. KPCA was used to analyse shape variability in trans-axial human brain medical images collected from University College Hospital Ibadan.

The hybridized segmentation model for the conventional Geometric Active Contour segmentation model and Kernel
Principal Component Analysis (KPCA) process was discussed in this section. The shape variability was extracted to form the feature space for medical image features to be extracted and segmented. These are the steps involved to derive the model;

(i) Let $\mathrm{T}=\{\mathrm{X} 1, \mathrm{X} 2, \ldots \ldots \ldots, \mathrm{XN}\}$ be a training set of registered medical images.

(ii) Selected feature was represented with high dimensional function (Signed distance function) and mean offset map was calculated using is closely related to this work in that Kernel Principal Component Analysis (KPCA) was used to model the shape variability and the distance between the shapes in the feature space and the initial constraint until the feature is extracted. Kernel Principal Component Analysis allows the extraction of nonlinear structure in the spaces of Signed distance functions and therefore performs better than linear Principal Component Analysis. The enhanced model (ENGAC) was formulated using Kernel Principal Component Analysis (KPCA) and Geometric active contour (GAC) segmentation model. KPCA was used to get shape variability within the Geometric active contour segmentation model. The training set of MRI and CT scan of tran-axial medical images were gotten from University College Hospital, Ibadan, Oyo state. The medical images were registered using appropriate registration method to pre-process the medical images [19].

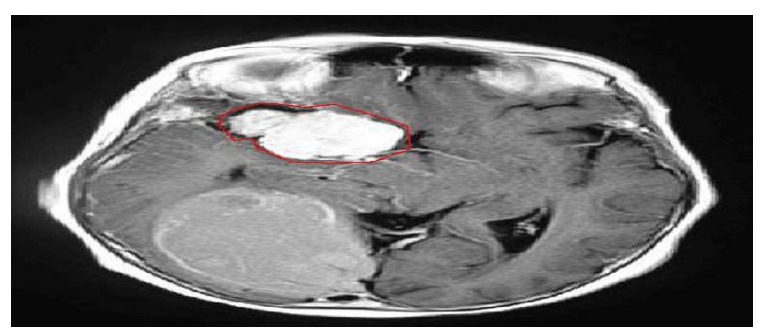

Fig 5 Gives the conceptual view of the enhanced geometric active contour model.

However, for the purpose of this work, the projection of the current Signed distance function was deliberately used to drive the evolution because of different object in the training set. The energy between the test shape and the shape in the Kernel PCA space need to be minimized so that outside of the initial constraint matches the background and the inside of the curve matches the boundary of feature to be extracted. Therefore the minimization of $\mathrm{E}_{\text {energy }}$ for any arbitrary contour results in the deformation of the contour towards a familiar shape.

An enhanced Geometric Active Contour model (ENGAC) that produced better segmentation results over the Geometric Active Contour (GAC) model is presented. Kernel Principal Component Analysis using Polynomial kernel function was used to get the shape variability before segmentation. This enhanced the model to accurately segment the selected feature or ROI (region of interest) and the model was validated by segmenting caudate nucleus and tumor from trans-axial Human Brain. 


\section{IMAGE SEGMENTATION BASED ON SOFTCOMPUTING}

\subsection{Supervised Segmentation}

With supervised classification, we identify examples of the Information classes (i.e., land cover type) of interest in the image. These are called "training sites". The image processing software system is then used to develop a statistical characterization of the reflectance for each information class. Supervised segmentation typically operate under one of the three paradigms for guidance:

a) Specification of parts of the boundary of the desired object or a nearby complete boundary that evolves to the desired boundary, as in active contours.

b) Specification of a small set of pixels belonging to the desired object and(possibly) a set of pixels belonging to the background, as in region growing, graph cut, and random walker.

c) Specification of starting and ending points along the target object's boundary and a minimal path approach that connect these points with a contour. The contour snaps to the desired boundary and wraps around the object, as in live wire" and intelligent scissors.

\subsection{Unsupervised Segmentation:}

This method in essence reverses the supervised classification process. Spatial/spectral classes are grouped first, based solely on the numerical information in the data, and are then matched by the analyst to information classes (if possible). Programs, called clustering algorithms, are used to determine the natural groupings or structures in the data.

Unsupervised classification is a method which examines a large number of unknown pixels and divides into a number of classed based on natural groupings present in the image values. unlike supervised classification, unsupervised classification does not require analyst-specified training data. The basic premise is that values within a given cover type should be close together in the measurement space (i.e. have similar gray levels), whereas data in different classes should be comparatively well separated (i.e. have very different gray levels) (PCI, 1997; Lillesand and Kiefer, 1994; Eastman, 1995)

The classes that result from unsupervised classification are spectral classed which based on natural groupings of the image values, the identity of the spectral class will not be initially known, must compare classified data to some form of reference data (such as larger scale imagery, maps, or site visits) to determine the identity and informational values of the spectral classes. Thus, in the supervised approach, to define useful information categories and then examine their spectral separability; in the unsupervised approach the computer determines spectrally separable class, and then define their information value. (PCI, 1997; Lillesand and Kiefer, 1994)

Unsupervised classification is becoming increasingly popular in agencies involved in long term GIS database maintenance. The reason is that there are now systems that use clustering procedures that are extremely fast and require little in the nature of operational parameters. Thus it is becoming possible to train GIS analysis with only a general familiarity with remote sensing to undertake classifications that meet typical map accuracy standards. With suitable ground truth accuracy assessment procedures, this tool can provide a remarkably rapid means of producing quality land cover data on a continuing basis.

For the last three decades, there have been two major groups of researchers in the field of algorithmic development for real systems; the first group believes that such development can only be done by using conventional mathematical and probabilistic techniques, Where as the second group emphasizes that there are other methods of applying mathematical knowledge that need not be that restrictive in terms of boundaries and samples.

An Enhanced Implementation of Brain Tumor Detection Using Segmentation Based on Soft Computing, a new unsupervised learning Optimization algorithms such as SOM are implemented to extract the suspicious region in the Segmentation of MRI Brain tumor. The textural features can be extracted from the suspicious region to classify them into benign or malign .This paper describes segmentation method consisting of two phases. In the first phase, the MRI brain image is acquired from patient's database. In that film artifact and noise are removed. After that Hierarchical Self Organizing Map (HSOM) is applied for image segmentation. The HSOM is the extension of the conventional self organizing map used to classify the image row by row. In this lowest level of weight vector, a higher value of tumor pixels, computation speed is achieved by the HSOM with vector quantization [9].

\section{CONCLUSIONS}

In the present work we have presented model-based segmentation with four types of comparative method, which are already the existing model-based segmentation methods and also in softcomputing is that here we proceed by using the well formulated active contour framework. Evaluation in comparison with manual segmentation has shown a good accuracy of our segmentation model. Landmark-based registration requires an expert user interaction for selecting the landmarks and this takes a considerable amount of time. The suggested object-based approach over-come this problem and resulting registration seems very close to the landmark-based one. For the 3D colonography segmentation, the shape of the colon sometimes is too complicated for tube-shaped model, or even a bendable tube-shaed to represent. This method has difficulties when the colon's shape changes rapidly [7] 


\section{FUTURE WORK}

The 3D segmentation is formulated in a non- parametric tracking frame work. Those experimentation results are also performing well. But those have also some limitations. In future the system should be improved by adapting more segmentation algorithm to suit the different medical image segmentation.

\section{REFERENCES}

[1]. Bessaud, Jean-Christophe et. al, "The Visible Human Slice Sequence Animation Web Server", Proc. The 3rd Visible Human Project Conference, Bethesda, Maryland, USA 2000

[2]. Gerlach, Sebastian. et al., "The Real -Time Interactive Visible Human Navigator", Proc. The 3rd Visible Human Project Conference, Bethesda, Maryland, USA, 2000

[3]. Yuh-Jye Chang, Paul Coddington and Karlie Hutchens, "Viewing the Visible Human using Java and the Web", Proc. of Asia Pacific Web (APWeb) '98,Beijing, Sept 1998, eds. Y. Yang et al., (International Academic Publishers,1998).

[4]. P.Gingins, P.Kalra, P. Beylot, N.Magnenat-Thalmann, J.Fasel, "Using VHD to build a comprehensive human model", Proc. The Visible Human Project Conference, Bethesda, Maryland, USA, 1996

[5]. Cootes, T., Taylor, C., Cooper, D., Graham, J.: Active shape models - their training and application. Computer Vision and Image Understanding 61 (1995) 38-59

[6]. Frey, B., Jojic, N.: Transformed component analysis: Joint estimation of spatial transformations and image components. In: IEEE Int'l Conf. on Computer Vision. (1999)

[7]. Qian, Z., Huang, X., Metaxas, D., Axel, L.: Robust segmentation of $4 \mathrm{~d}$ cardiac mri-tagged images via spatiotemporal propagation. In: Proceedings of SPIE Medical Imaging: Physiology, Function, and Structure from Medical Images. Volume 5746. (2005) 580-591

[8]. Krishna Kant Singh, Akansha Singh, Deptt. Of Electronics \& Communication, Hermes Engineering College ,Roorkee , India Deptt. Of Information Technology AKGEC,Ghaziabad, India" A Study Of Image Segmentation Algorithms For Different Types Of Images" IJCSI International Journal of Computer Science Issues, Vol. 7, Issue 5,September 2010.

[9]. Karnan.M,Logeswari.T,Selvanayaki.K,:'Introduction to detection of Automatic brain tumor through MRI" , Proceedings of National Conference and Wokshop on Soft and Intelligent Computing,23- 25,jan 2008.

[10]. P.Lakshmi Devi, Associate professor, Dept of ECE, AITS Rajampet, Kadapa. AP , INDIA S.Varadarajan, Associate professor, Dept of ECE, SVU College of Engg, Tirupathi. AP INDIA "Image Segmentation and Techniques: A Review" Proceedings of the International Journal of Advanced Research and Technology, IJART, Vol. 1 Issue 2, 2011,118-127

[11]. D.J. Withey and Z.J. Koles,Department of Electrical and Computer Engineering , Department of Biomedical Engineering, University of Alberta, Edmonton, Alberta, CANADA "Medical Image Segmentation: Methods and Software" Proceedings of the NFSI and ICFBI 2007, Hangzou, China, October 12-14, 2007
[12]. Prof. Samir Kumar Bandyopadhyay , Dept. of Computer Sc. \& Engineering, University of Calcutta ,Kolkata, India

"A Survey on Brain Image Segmentation Methods" Proceedings of the Journal of Global Research in Computer Science, Volume 2, No. 2, February 2011

[13]. Sukhmanpreet Singh, Deepa Verma, Arun Kumar, Rekha "Image Segmentation Using Soft Computing"

[14]. N. Senthilkumaran and R. Rajesh ,School of Computer Science and Engineering, Bharathiar University, Coimbatore , India "Edge Detection Techniques for Image Segmentation - A Survey of Soft Computing Approaches" Proceedings of the International Journal of Recent Trends in Engineering, Vol. 1, No. 2, May 2009

[15]. A. Suneel kumar Reddy, J. V. K. Ratnam ,Department of Electronics and Communication Engineering "Image Segmentation using Texture Feature" Proceedings of International Journal of Electronics and Computer Science Engineering.

[16]. Dr. Leow Wee Kheng (Associate Professor) and Ding Feng, School of Computing, National University of Singapore July 2006 "Segmentation of Bone Structures inX-ray Images"

[17]. Biplab Banerjee, Tanusree Bhattacharjee \& Nirmalya Chowdhury "Color Image Segmentation Technique Using Natural Grouping of Pixels" Proceedings of the International Journal of Image Processing(IJIP), Volume(4): Issue(4)

[18]. Ali Kermani, Ahmad Ayatollahi, Ahmad Mirzaei, Mohammad Barekatain, School of Electrical Engineering, Iran University of Science and Technology, Tehran, Iran "Medical ultrasound image segmentation by modified local histogram range image method" Proceedings of the J. Biomedical Science and Engineering, 2010, 3, 1078-1084

[19]. Ajala F.A and Emuoyibofarhe O.J. (2012): Performance evaluation of Enhanced Geometric active contour model (ENGAC) and Geometric active contour model (GAC) for medical image segmentation.Computer Engineering and Intelligent Systems, 3(3):78-86. 